\title{
FECUNDIDAD, MIGRACIÓN Y ASPIRACIONES DE MOVILIDAD SOCIAL EN LOS PAÍSES EN DESARROLLO: SUGERENCIAS PARA INVESTIGACIÓN *
}

\author{
ROBERT H. WELLER \\ Universidad Brown \\ $\mathrm{y}$ \\ JoHN J. MAcrsco JR. \\ Centro Latinoamericano de Demografía
}

\section{INTRODUCción}

EsTE es un ejercicio heurístico en el cual examinamos niveles de fecundidad alternativos entre migrantes hacia San Juan provenientes de áreas no metropolitanas de Puerto Rico y residentes no migrantes de San Juan entre 1955 y 1960 . En vista de la fecundidad marcadamente mayor en las zonas no metropolitanas de Puerto Rico en 1960, y la investigación previa de Goldberg y Duncan con base en datos de los Estados Unidos, se esperaría una fecundidad mayor entre los inmigrantes que entre los no migrantes. Esta esperanza no se cumple. Más aún, la ausencia de tal esperanza no es función de la selectividad superior de los migrantes con respecto a las características educativas y ocupacionales de la cónyuge. Por lo tanto, deben operar otra clase de variables además de los antecedentes no metropolitanos y las características socioeconómicas. Nuestra conclusión consiste en proponer el estudio de una de tales variables; las aspiraciones relativas de mo. vilidad social ascendente entre los migrantes y los inmigrantes y considerar el grado en que esto pueda generalizarse a los países en desarrollo en general.

Muchos países poco desarrollados experimentan tasas extremas de urbanización y tasas de crecimiento urbano aún mayores. Al hablar de "urbanización" nos referimos a un aumento de la proporción de

* El título original de este trabajo es "Migration, Aspirations for Social Mobility, and Fertility in Developing Countries: Sugestions for Further Research". La traducción es de A. García Rocha.

1 Revisión del trabajo presentado en 1969 en la reunión anual de la Asociación Americana para el Avance de la Ciencia. Parte de esta investigación ha sido patrocinada por el fondo del Servicio de Salud Pública de los Estados Unidos número CHO0272-02; Fundación Ford, donativo número 63-75; y Fundación Nacional de la Ciencia, donativo número GS-3078. Expresamos nuestro agradecimiento a las numerosas personas que leyeron versiones anteriores de este manuscrito y quienes hicieron útiles comentarios. 
habitantes de un país que reside en localidades urbanas. "Crecimiento urbano" se refiere a un aumento del número de personas que viven en localidades urbanas. Dada una definición constante de fronteras "urbanas" y de fronteras políticas fijas, el crecimiento urbano puede presentarse debido a la inmigración neta $\mathrm{y} / \mathrm{o}$ a una tasa de crecimiento natural positiva. En contraste, la urbanización puede presentarse a través de la migración rural urbana y/o una tasa de crecimiento natural mayor en las áreas urbanas que en las rurales. El crecimiento urbano puede presentarse en ausencia de urbanización $\mathrm{y}$, en una población cuyo tamaño disminuye, aquella última puede presentarse ante la ausencia de crecimiento urbano. Sin embargo, estos procesos normalmente ocurren juntos. Árnbos conceptos deberían distinguirse de "urbanismo", el cual ha definido Wirth (1938) como "aquel complejo de facetas que constituyen el modo característico de vida en las ciudades..." Mientras que exista desacuerdo sobre el significado preciso de urbanismo, resulta claro que este concepto representa un intento por introducir algunas de las consecuencias sociológicas y psicológicas de los patrones residenciales y que estas consecuencias tienen implicaciones en la fecundidad.

Históricamente, las tasas de fecundidad han sido menores en las áreas urbanas que en las rurales y por lo general se ha supuesto que la migración rural urbana ha sido la causa principal de la urbanización. La primacía de la migración como determinante del crecimiento urbano resulta menos clara. Varios observadores han declarado que la inmigración explica casi el $50 \%$ del crecimiento urbano en muchos países latinoamericanos (cf. Wingo, 1967; NU, 1961, 110; y Orbe, Caicedo y Costales, $1961: 265)$. Arriaga (1968) argumenta que una tasa de crecimiento natural elevada en las áreas urbanas -debida a niveles de fecundidad urbana relativamente altos y a niveles de mortalidad bajos, hechos posibles por la importación de tecnología médica, los programas de salud pública y su concentración en las ciudades- es la causa principal del crecimiento urbano de América Latina.

Sin embargo, Arriaga no atribuye importancia suficiente al hecho de que el crecimiento natural urbano es la suma ponderada del crecimiento natural de los inmigrantes y los no migrantes. De este modo,

$$
\text { Cracimiento urbano }=\text { Migración neta }+\sum_{i=1}^{n} W_{i}\left(B_{i}-D_{i}\right)
$$

donde $W$ se refiere a la proporción de la población que es urbana de primera generación, urbana de segunda generación ( $\mathrm{y}$ así sucesivamente), $B-D$ es el crecimiento natural de ese grupo, y $n$ el número de generaciones presente.

El significado de esta consideración, al comparar el papel de la inmigración frente al crecimiento natural como agente que produce crecimiento urbano, queda ilustrado en un estudio reciente con datos brasileños que muestra que las mujeres inmigrantes explican el 45-50 \% de todos los embarazos en el estado de Guanabara (Martine, 1969:200). Más aún, los niños nacidos de inmigrantes una vez que han migrado a la ciudad están clasificados como nativos urbanos, e inclusive la distinción entre nacimiento de nativos y nacimientos de inmigrantes podría conducir a una subdeclaración significativa del 
papel de la migración en el crecimiento urbano. Así, Browning y Feindt (1968:189) informan que el 52\% de los "nativos por nacimiento" en su muestra de 1965 de hombres de 21-60 años de edad que viven en Monterrey, México, declaró que ambos padres habian nacido fuera de Monterrey. (Para un análisis del grado en que la migración afecta el crecimiento urbano en América Latina, véase Weller, Macisco y Martine, 1971.)

Así, parece evidente que para entender a fondo el proceso por el cual el crecimiento urbano está ocurriendo en las áreas menos desarrolladas, debemos considerar el grado en que difiere la fecundidad de los migrantes y la de los no migrantes urbanos, a qué grado los inmigrantes tienen sus hijos antes y/o después de llegar a la ciudad y si la generación con residencia urbana afecta los niveles de fecundidad. Debido a las limitaciones de nuestros datos, en el trabajo presente podemos considerar empíricamente sólo el primero de estos factores. Sin embargo, hacemos hincapié en la importancia de los últimos factores y esperamos que, al establecer en forma explícita las cuestiones, estimularemos la investigación futura por estas líneas.

\section{FECUNDIDAD DE MigRaNTES Y No MigRantes}

Aunque por lo general sucede que la fecundidad rural es mayor que la correspondiente a las áreas urbanas (y el tamaño de la comunidad guarda correlación inversa con la fecundidad), la cuestión de si los migrantes rural-urbanos tienen fecundidad mayor que los no migrantes urbanos no es la misma en todas las sociedades. En general, la investigación en las zonas relativamente industrializadas ha indicado que los inmigrantes urbanos tienen fecundidad mayor que sus contrapartes nacidos en la ciudad. (Thomas, 1938:320-322; Goldberg, 1959; y Duncan, 1965.) Sin embargo, en un análisis de datos recogidos en el estudio de Indianápolis, Kiser (1938) concluyó que la fecundidad marital de los inmigrantes nacidos en el medio urbano, de raza blanca, que migraron antes de casarse, no fue superior a la observada entre las personas nacidas en la ciudad en edad y posición social comparables. Esto sugiere que la exposición al ambiente urbano durante el periodo reproductivo conduce la socialización hacia las normas y prácticas urbanas que a su vez conducen a una fecundidad menor. Así, se esperaría que mientras mayor sea la exposición, más parecida será la fecundidad de los inmigrantes a la de los nativos urbanos. Los hallazgos de Kiser sugieren también la importancia potencial de la "etapa de llegada", es decir, el punto en la vida o en el ciclo familiar en el cual ocurrió esta exposición.

Los estudios que comparan los niveles relativos de los migrantes y los no migrantes en América Latina no han sido concluyentes. Germani (1961), Hutchinson (1961), Martine (1969), y Miró y Rath (1965) informan que los inmigrantes tienen fecundidad mayor a la de los no migrantes. Además, Germani (1961-217) informa que los migrantes más recientes tienen más hijos que los menos recientes. Sin embargo, Zárate (1967) informa que la fecundidad no manifiesta una relación clara con la duración de la residencia en Monterrey; y los datos reco- 
gidos en Lima, Perú, no muestran diferencias sustanciales entre la fecundidad de los migrantes a Lima y los nativos de estas ciudades cuando se controla la edad (Alers y Appelbaum, 1968:32). Tabah y Samuel $(1968: 282)$ no informan de diferencias de la fecundidad entre los inmigrantes a Santiago y los residentes nacidos en ciudad, y datos de Santiago publicados más recientemente conducen a la conclusión de que los inmigrantes tienen fecundidad menor en todas las edades menores de 40 años (Elizaga, 1966). Más aún, varios estudios de datos sobre Puerto Rico indican que la migración y la fecundidad están asociados en forma negativa (Hatt, 1952: 310-314; Macisco, 1965; y Myers y Morris, 1966).

Tal vez estos informes divergentes son función de diferencias en el diseño del estudio, las muestras estudiadas y las definiciones operacionales de la migración y la fecundidad. La investigación sobre la fecundidad y la migración rural urbana ha estado caracterizada generalmente por falta de control de las diferencias en las estructuras por edad, edad al matrimonio, variaciones en el espaciamiento de los hijos, la edad en el momento de la migración, y de si la migración ha ocurrido en uno o varios pasos.

Es probable también que la relación entre migración y fecundidad varíe de un ambiente social a otro y que sea producida primordialmente por la migración selectiva. Así, las variables en las cuales ocurre la selección, la intensidad de ésta, el si favorece una mayor o menor fecundidad entre migrantes, y las condiciones bajo las cuales varían estos factores, son elementos que asumen la mayor importancia en cualquier esquema conceptual de la relación entre urbanización, migración y fecundidad.

Existen varios mecanismos causales alternativos según los cuales los inmigrantes y los no migrantes urbanos podrian presentar diferencias de fecundidad. Primero, supóngase que es exacta la noción de medio urbano y su influencia sobre el comportamiento demográfico individual, según se describe en las explicaciones acostumbradas sobre la transición demográfica. Esto es, el medio urbano es tal que los grandes costos sociales, sicológicos y económicos se atribuyen al hecho de tener grandes familias. Esto debería crear una motivación generalmente mayor en el sentido de tener familias más pequeñas que lo que ocurriría ante la ausencia (o el debilitamiento) de estas restricciones. En un caso como éste un migrante hacia la ciudad sería gradualmente absorbido o socializado por la cultura de la ciudad y eventualmente exhibiría un comportamiento similar al del nacido en tal medio.2 Supuestamente, mientras mayor sea la duración de la residencia en la ciudad y menor la edad al iniciarse la residencia, mayor será la similitud entre inmigrantes y nativos y el migrante parecerá más "urbano". Más aún, mientras mayor sea el tamaño de la comunidad de origen, mayor será la similitud entre los inmigrantes y los

2 Además de las normas relacionadas con un tamaño menor de la familia, otro indicador de asimilación en el modo de vida urbano es la participación en organizaciones formales y asociaciones voluntarias. Cuando se comparan los niveles de participación de migrantes y no migrantes, lo resultados son paralelos y congruentes con las ideas expresadas en esta sección (C. Zimmer, 1955; Zimmer, 1956; y Germani, 1961: 224225). 
nativos de la ciudad. Uno podría también desear tomar en cuenta el hecho de que la migración fue directa o por etapas, postulando que -bajo el supuesto de que lc demás permanece igual- mientras mayor sea el número de pasos en una cadena migratoria particular, mayor será el parecido entre migrantes y no migrantes, ambos residentes de la ciudad. Por último, el proceso de socialización en el medio de vida urbano puede depender de los patrones residenciales dentro de la ciudad. Por ejemplo, Abu-Lughod (1961) informa que en El Cairo existen numerosas concentraciones de migrantes de poblados particulares dentro de pequeñas subsecciones de la ciudad. Uno puede esperar que la asimilación disminuya según el grado al cual los inmigrantes rurales establezcan "poblados" dentro de la localidad urbana. De este modo, cualesquiera diferencias en el agregado entre la fecundidad de los inmigrantes y los nativos urbanos sería en parte función positiva del grado al cual están presentes proporciones de migrantes cuya exposición al medio urbano es insuficiente para ocasionar la asimila. ción completa.

Una explicación alternativa pone mayor énfasis en características algo diferentes de los inmigrantes. Puede ser que éstos sean seleccionados en cuanto a características que están firmemente relacionadas con la fecundidad y que cualquier diferencia de la fecundidad exista debido a que los inmigrantes poseen estas características en un grado mayor (o menor) que los nacidos en la ciudad. Si esta línea de razonamiento fuera correcta, el control de estas características resultaría en la desaparición de las diferencias de fecundidad.

Las variables socioeconómicas han recibido énfasis especial como una explicación potencial de las diferencias de fecundidad según el status migratorio. Así, una vez observado que la migración guarda relación inversa con la fecundidad, a lo largo del período fértil, en el caso de las mujeres de Puerto Rico, Myers y Morris (1966:96) inquieren: "¿Tendrían sustancia estos hallazgos si se introdujeran controles de los niveles socioeconómico y educativo?".

El estudio presente persigue esta línea de investigación mediante el análisis de diferencias de fecundidad entre migrantes y no migrantes en el Arrea Estadística Metropolitana Estándar de San Juan (AMSJ) mientras que alternativamente mantiene constante el nivel educativo de la esposa y varias características ocupacionales de la esposa y el marido.

\section{FUENTE DE LOS DATOS}

Este estudio se basa en tabulaciones especiales del censo de 1960 de la Comunidad de Puerto Rico. Se consideran dos grupos, los no migrantes, que son personas que vivieron en el AMSJ tanto en 1955 como en 1960, y aquellos inmigrantes que declararon un lugar de residencia no metropolitano en 1955. Este análisis excluye a los emigrantes a San Juan de las dos áreas metropolitanas restantes de Ponce y Mayaguez y de los Estados Unidos del continente. El estado migratorio es el del marido y el análisis delimita a las mujeres casadas legalmente $\mathrm{y}$ en edades 14-44. La fecundidad se mide según el número de hijos nacidos. Así, estamos comparando la fecundidad de las esposas de 
inmigrantes provenientes de áreas no metropolitanas, con la fecundidad de sus contrapartes no migrantes residentes del lugar de destino. Las fallas de las diversas medidas de la migración y la fecundidad que se utilizan ya se han descrito previamente, ${ }^{3}$ pero deseamos hacer hincapié en que una proporción desconocida de inmigrantes vivió en un lugar urbano en el área no metropolitana en 1955, y que una proporción sustancial de los no migrantes nació probablemente en un área metropolitana y emigró a San Juan antes de 1955. Una proporción desconocida de la fecundidad de estos migrantes recientes ocurrió antes de la migración. Las variables socioeconómicas se basan en la situación de la persona en 1960 y no en el momento de la migración. Más todavía, advertimos que las dicotomías simples entre migrantes y no migrantes pueden ocultar diferencias en la posición socioeconómica que pueden variar según la duración de la residencia en el área metropolitana y la edad a la cual la persona emigró (cf. Browning y Feindt, 1968).

Nos gustaría comentar también sobre la magnitud de las diferencias de fecundidad entre los inmigrantes y los no migrantes. Las mujeres legalmente casadas menores de 45 años y esposas de inmigrantes tienen un promedio de 2.7 hijos nacidos, mientras que las esposas de los no migrantes tienen un promedio de 3.1. El análisis de los datos del censo de 1960 revela que la desviación estándar de los niños nacidos de mujeres alguna vez casadas de las edades 15-44, residentes de San Juan, es de 2.45 (Oficina del Censo de los Estados Unidos, 1962, cuadro 94). Aunque la inclusión de viudas, divorciadas y separadas indudablemente eleva la estimación de la varianza, resulta claro que la diferencia real entre los inmigrantes es primordialmente de interés teórico. Desde luego que esta diferencia observada es estadísticamente significativa.

Más aún, dado el gran número de casos en nuestro análisis, este patrón persiste en muchos cruzamientos tabulados y el modelo de los países industrializados sugiere que los inmigrantes deberían tener una fecundidad mayor que la de los no migrantes urbanos. Así, subsiste la cuestión de si ello se debe a diferencias en las características socioeconómicas. ¿Tienen los migrantes características que en otras circunstancias serían favorables a que tuvieran fecundidad menor, y compensa esta diferencia su antecedente rural en cuanto a la fecundidad?

\section{Migración, EDUCAción y FECUNDIDAD}

La relación inversa entre educación y fecundidad en América Latina está bien establecida, y llevó a Miró y Mertens (1968:106) a declarar: "Ninguna otra variable socioeconómica muestra una relación tan clara con la fecundidad como la educación". Más aún, respecto de Puerto Rico, Stycos (1967) hace notar que las mujeres con cero educación tienen 3.3 más hijos que aquellas que tienen al menos un año de educación universitaria. ${ }^{4}$

Los estudios sobre la relación entre educación y migración han sido menos definitivos. Los estudios realizados en México (Browning y

3 Cf. Macisco, Bouvier y Renzi, 1969; y Macisco, Bouvier y Weller, 1970.

4 Ver también Bouvier y Macisco (1968); Godley (1968), y Dinkel (1967). 
Feindt, 1969) y en Formosa (Speare, 1969) indican que los migrantes hacia áreas metropolitanas de Monterrey y Taichung tienden a tener mayor educación que los que permanecen en el punto de origen. Los datos de los Estados Unidos muestran también una asociación positiva entre la migración rural metropclitana y el nivel educativo (Folger y Nam, 1967).

En relación con las comparaciones hechas en el área de destino, algunos datos de los Estados Unidos indican que los inmigrantes provenientes de áreas rurales tienen niveles educativos algo menores que los que permanecen en las metrópolis (cf. Bogue y Hagood, 1953; gráficas 9, 10 y 11). Sin embargo, un examen de los datos del censo de 1960 revela que los inmigrantes tuvieron alrededor de dos veces la

\section{Cuadro 1}

Puerto Rico: Años de escuela terminados. Mujeres legalmente casadas con MARIDO PRESENTE, SEGÚN LA EDAD DE LA ESPOSA Y LA POSICIÓN MIGRATORTA DEL MARIDO, 1960

\begin{tabular}{|c|c|c|c|c|c|}
\hline \multirow{2}{*}{$\begin{array}{c}\text { Edad y educación } \\
\text { de las mujeres }\end{array}$} & \multirow{2}{*}{\multicolumn{2}{|c|}{$\frac{\text { No migrantes }}{\text { Numero }}$}} & \multicolumn{3}{|c|}{ Migrantes } \\
\hline & & & Núme ro & & $\%$ \\
\hline \multicolumn{6}{|l|}{$14-24$} \\
\hline $\begin{array}{l}\text { Sin educación } \\
\text { Elemental }(1-8) \\
\text { Secundaria }(9-12) \\
\text { Universidad }(13+)\end{array}$ & $\begin{array}{r}88 \\
1106 \\
863 \\
242 \\
\end{array}$ & $\begin{array}{r}3.8 \\
48.2 \\
37.5 \\
10.5 \\
\end{array}$ & $\begin{array}{r}\epsilon \\
112 \\
116 \\
45 \\
\end{array}$ & & $\begin{array}{r}2.2 \\
40.1 \\
41.6 \\
16.1 \\
\end{array}$ \\
\hline $\begin{array}{l}\text { Total } \\
\text { Mediana }\end{array}$ & 2299 & 100.0 & 279 & 8.7 & 100.0 \\
\hline \multicolumn{6}{|l|}{$25-34$} \\
\hline $\begin{array}{l}\text { Sin educación } \\
\text { Elemental } \\
\text { Secundaria } \\
\text { Universidad }\end{array}$ & $\begin{array}{ll} & 292 \\
2 & 355 \\
1 & 720 \\
1 & 035 \\
\end{array}$ & $\begin{array}{r}5.4 \\
43.6 \\
31.8 \\
19.2 \\
\end{array}$ & $\begin{array}{r}20 \\
157 \\
117 \\
131 \\
\end{array}$ & & $\begin{array}{r}4.7 \\
36.9 \\
27.6 \\
30.8 \\
\end{array}$ \\
\hline $\begin{array}{l}\text { Total } \\
\text { Mediana }\end{array}$ & 5402 & 100.0 & 425 & 9.2 & 100.0 \\
\hline \multicolumn{6}{|l|}{$35-44$} \\
\hline $\begin{array}{l}\text { Sin educación } \\
\text { Elemental } \\
\text { Secundaria } \\
\text { Universidad }\end{array}$ & $\begin{array}{ll} & 348 \\
2 & 742 \\
1 & 101 \\
708 \\
\end{array}$ & $\begin{array}{r}7.1 \\
56.0 \\
22.5 \\
14.5 \\
\end{array}$ & $\begin{array}{r}23 \\
147 \\
59 \\
46 \\
\end{array}$ & & $\begin{array}{r}8.3 \\
53.5 \\
21.5 \\
16.7 \\
\end{array}$ \\
\hline $\begin{array}{l}\text { Total } \\
\text { Mediana }\end{array}$ & 4899 & 100.1 & 275 & 6.3 & 100.0 \\
\hline \multicolumn{6}{|l|}{$\begin{array}{l}\text { Todas las } \\
\text { mujeres de 14-44 }\end{array}$} \\
\hline $\begin{array}{l}\text { Sin educación } \\
\text { Elemental } \\
\text { Secundaria } \\
\text { Universidad }\end{array}$ & $\begin{array}{l}728 \\
6203 \\
3684 \\
1985 \\
\end{array}$ & $\begin{array}{r}5.8 \\
49.2 \\
29.2 \\
15.8 \\
\end{array}$ & $\begin{array}{r}49 \\
416 \\
292 \\
222 \\
\end{array}$ & & $\begin{array}{r}5.0 \\
42.5 \\
29.8 \\
22.8 \\
\end{array}$ \\
\hline $\begin{array}{l}\text { Total } \\
\text { Mediana }\end{array}$ & 12600 & 100.0 & 979 & 9.3 & 100.1 \\
\hline
\end{tabular}

Fuente: Tabulaciones especiales inéditas preparadas para este proyecto por la Oficina de Censos de los Estados Unidos a partir de datos facilitados por la Junta de Planificación de Puerto Rico. Ver también Macisco, Bouvier y Renzi, op. cit., p. 173. 
proporción de universitarios graduados que la población residente en las grandes ciudades, y cerca de la misma proporción que los emigrantes hacia otras áreas metropolitanas (Folger y Nam, 1967: 182); éste es un patrón que podría muy bien ser producto de las diferencias de edad.

Con respecto a El Salvador, Ducoff (1962) concluyé que existe se. lección en ambos extremos de la escala educativa, es decir, "una concentración mayor entre los migrantes con muy poca escolaridad así como entre las personas con niveles educativos elevados". Los datos de Monterrey y Santiago indican que los inmigrantes tienen menos educación que los nativos (cf. Browning y Feindt, 1968; Elizaga, 1966; y Aers y Appelbaum, 1968).

De este modo, hemos usado los datos de San Juan para dar respuesta a dos cuestiones:

1. ¿Las esposas de los inmigrantes tienen niveles de educación diferentes a los de las esposas de los residentes metropolitanos clasificados como no migrantes?

2. Si es así ¿produce el control de estas diferencias una situación según la cual las personas con antecedentes no metropolitanos tienen mayor fecundidad que los no migrantes?

En el cuadro 1 puede verse que las cónyuges de los emigrantes han alcanzado niveles educativos algo superiores a las de los no migrantes. Cuando se controla la edad, los niveles son idénticos en el grupo de edad mayor y entre las categorías más jóvenes existe una

\section{Cuadro 2}

Puerto RioO: Hijos Nacidos vivos POR CADA 1000 MUJERES CASADAS LEGALMENTE Y CON ESPOSO PRESENTE, SEGT'N EDAD Y EDUCACIÓN DE LA ESPOSA Y POSICIÓN MIGRATORIA DEL MARIDO, 1960

\begin{tabular}{|c|c|c|c|c|}
\hline & \multicolumn{3}{|c|}{ Edad de la esposa } & \multirow{2}{*}{$\frac{\text { Sot } i 1]}{14-44}$} \\
\hline & $\overline{14-24}$ & $25-34$ & $35-44$ & \\
\hline \multicolumn{5}{|l|}{ Sin educación } \\
\hline $\begin{array}{l}\text { Esposa de mirrante } \\
\text { Esposa de no migrante } \\
\text { Cociente } \mathrm{W} / \mathrm{MK}\end{array}$ & $3 \stackrel{x}{364}$ & $\begin{array}{rl}4 & 050 \\
4894 \\
.83\end{array}$ & $\begin{array}{rl}4 & 783 \\
5 & 543 \\
.86\end{array}$ & $\begin{array}{r}4339 \\
5099 \\
.86\end{array}$ \\
\hline \multicolumn{5}{|l|}{ Elemental } \\
\hline $\begin{array}{l}\text { Espoca de migrante } \\
\text { Eeposa de no migrante } \\
\text { cociente } \mathrm{M} / \mathrm{M}\end{array}$ & $\begin{array}{r}1964 \\
2162 \\
.90\end{array}$ & $\begin{array}{r}3739 \\
3707 \\
1.01\end{array}$ & $\begin{array}{r}4939 \\
4418 \\
1.12\end{array}$ & $\begin{array}{r}3685 \\
3746 \\
\quad .98\end{array}$ \\
\hline \multicolumn{5}{|l|}{ Secundaria } \\
\hline $\begin{array}{l}\text { Esposa de mierante } \\
\text { Esposa de no migrante } \\
\text { Cociente } M / M\end{array}$ & $\begin{array}{rl}1 & 129 \\
1 & 441 \\
\quad .78\end{array}$ & $\begin{array}{l}2205 \\
2313 \\
\quad .95\end{array}$ & $\begin{array}{rl}2 & 458 \\
2 & 597 \\
.95\end{array}$ & $\begin{array}{rl}1 & 829 \\
2 & 194 \\
\quad .83\end{array}$ \\
\hline \multicolumn{5}{|l|}{ Univeroidad } \\
\hline $\begin{array}{l}\text { Esposa de migrante } \\
\text { Esposa de no migrante } \\
\text { Cociente } \mathrm{K} / \mathrm{m}\end{array}$ & $\begin{array}{l}756 \\
901 \\
.84\end{array}$ & $\begin{array}{r}1802 \\
2064 \\
.87\end{array}$ & $\begin{array}{r}2457 \\
2 \quad 367 \\
1.04\end{array}$ & $\begin{array}{rl}1 & 726 \\
2 & 030 \\
\quad .85\end{array}$ \\
\hline \multicolumn{5}{|c|}{ Todos los niveles educativos } \\
\hline $\begin{array}{l}\text { Esposa de migrante } \\
\text { Esposa de } \mathrm{nom} \text { migrante } \\
\text { Cociente } \mathrm{M} / \mathrm{Mm}\end{array}$ & $\begin{array}{r}1430 \\
1805 \\
.79\end{array}$ & $\begin{array}{r}2734 \\
3013 \\
.91\end{array}$ & $\begin{array}{r}3.978 \\
3792 \\
1.04\end{array}$ & $\begin{array}{r}2712 \\
3.005 \\
.88\end{array}$ \\
\hline
\end{tabular}

Fuente: Derivado de Macisco, Bouvier y Renzi, op. cit., p. 175; el número de casos aparece en el cuadro 1.

* Menos de 15 casos. 
diferencia de alrededor de un año, en años promedio de escolaridad.

En el cuadro 2 y en la gráfica 1 puede verse que existe la fuerte relación negativa esperada entre la educación y la fecundidad. Cuando se controla la educación, la diferencia en la fecundidad entre las esposas de inmigrantes y de no migrantes se reduce considerablemente. Sin embargo, la noción occidental de antecedente no metropolitano nos conduciría a esperar una fecundidad mayor entre los inmigrantes. Éste no es el caso y parece no haber interacciones.

\section{MigRación, POSICIÓN OCUPACIONAL Y FECUNDIDAD}

Otra variable que podría ayudar a explicar por qué las cónyuges de los inmigrantes no tienen fecundidad mayor que las de los no migrantes es la participación en la fuerza de trabajo. Por lo general se ha supuesto que, debido a que la gente emigra por razones económicas, los migrantes tienen mayor probabilidad que los no migrantes de pertenecer a la fuerza de trabajo. Basando sus conclusiones en un análisis de los datos del censo de 1960 de los Estados Unidos, Miller (1966) rechaza esta hipótesis entre los blancos pero la acepta para el

\section{Gráfica 1}

Puerto Rico: Hijos nacidos vivos de MUJeres legalmente Casadas CON CÓNYUGE PRESENTE, POR STATUS DEL MARIDO, POR EDAD

Y EDUCACIÓN DE LA ESPOSA, 1960

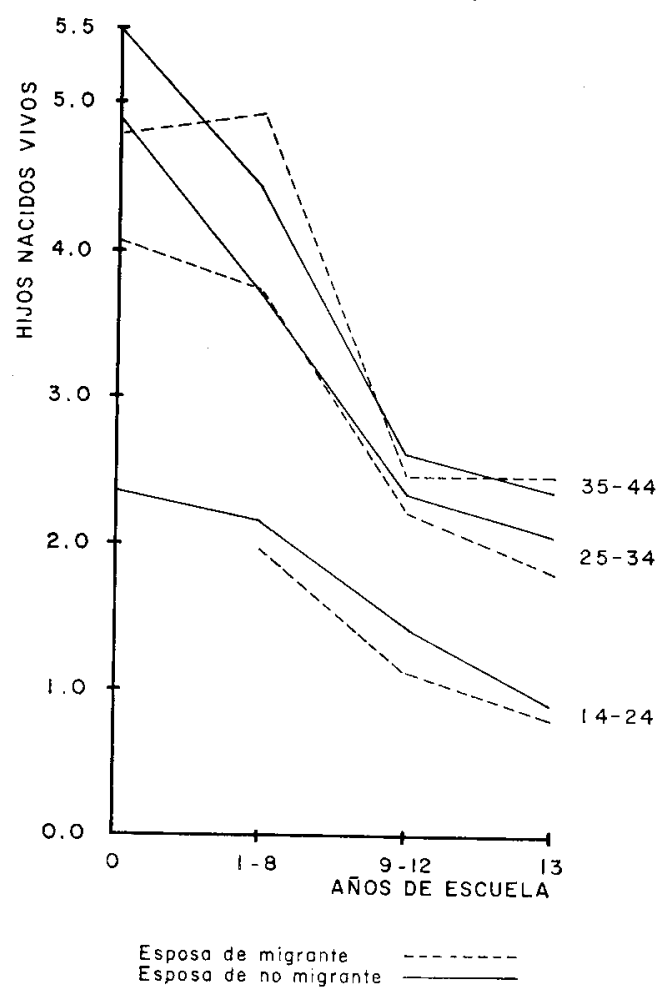


caso de la población de color. (En relación con una advertencia metodológica, cf. Masnick, 1968.) Los datos recolectados en Santiago (Elizaga, 1966) y Bombay (Zachariah, 1966) indican también tasas de participación en la fuerza de trabajo mayores entre los inmigrantes que entre los nativos metropolitanos.

La relación entre participación activa en la fuerza de trabajo femenina y la fecundidad está razonablemente establecida en Puerto Rico, donde las cónyuges que trabajan -en especial aquellas con puestos de oficina o de "cuello blanco"- acusan fecundidad menor que aquellas que no trabajan (Vázquez, 1964; y Weller, 1968).

Esto sugiere los postulados siguientes: las personas que emigran a San Juan tienen mayor probabilidad de pertenecer a la fuerza de trabajo y de tener cónyuges (femeninas) en la fuerza de trabajo. Esta mayor tasa de participación, en especial de las cónyuges, está asociada con una fecundidad menor. Por lo tanto, cuando hacemos comparaciones dentro de las categorías de fuerza de trabajo, debemos encontrar que los inmigrantes tienen fecundidad mayor que los no migrantes.

En los cuadros 3 y 4 puede verse que no hay asociación entre la posición migratoria del marido y sus características ocupacionales. Sin embargo, las cónyuges de los migrantes tienen una probabilidad algo mayor que las de los no migrantes de pertenecer a la fuerza de trabajo ${ }^{5}$ y de obtener ocupaciones de "cuello blanco". De este modo estos datos no dan apoyo al punto de vista común de que los inmigrantes con origen no metropolitano tienen relativamente menos educación y no tienen las habilidades para los empleos de "cuello blanco" requeridas en la economía metropolitana.

\section{Cuadro 3}

Puerto Rico: Participación en la fuerza de trabajo. Maridos y esposas SEGÍN EDAD DE LA ESPOSA Y POSICIÓN MIGRATORIA DEL MARIDO, ÁREA

Estadística Metropolitana Estándar de San Juan, 1960

\begin{tabular}{|c|c|c|c|c|c|}
\hline & Total & $\begin{array}{c}\text { El marido } \\
\text { trabajo }\end{array}$ & 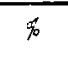 & $\begin{array}{c}\text { La esposa } \\
\text { trabaja }\end{array}$ & $\%$ \\
\hline \multicolumn{6}{|l|}{$\begin{array}{c}\text { No } \\
\text { migrantes }\end{array}$} \\
\hline $\begin{array}{l}14-24 \\
25-34 \\
35-44\end{array}$ & $\begin{array}{ll}2 & 303 \\
5 & 409 \\
4 & 911\end{array}$ & $\begin{array}{ll}2 & 163 \\
5 & 185 \\
4 & 597\end{array}$ & $\begin{array}{l}93.9 \\
95.9 \\
93.6\end{array}$ & $\begin{array}{l}489 \\
1616 \\
1396\end{array}$ & $\begin{array}{l}21.2 \\
29.9 \\
28.4\end{array}$ \\
\hline Total & 1262.3 & 11945 & 94.6 & 3501 & 27.7 \\
\hline \multicolumn{6}{|l|}{ Migrantes } \\
\hline $\begin{array}{l}14-24 \\
25-34 \\
35-44\end{array}$ & $\begin{array}{l}279 \\
426 \\
276\end{array}$ & $\begin{array}{l}263 \\
389 \\
254\end{array}$ & $\begin{array}{l}94.3 \\
91.3 \\
92.0\end{array}$ & $\begin{array}{r}74 \\
153 \\
81\end{array}$ & $\begin{array}{l}26.5 \\
35.9 \\
29.3\end{array}$ \\
\hline Total & 981 & 906 & 92.4 & 308 & 31.4 \\
\hline
\end{tabular}

Fuente: la misma del cuadro 1. Ver también Macisco, Bouvier y Weller, op. cit.

En los cuadros 5 y 6 se compara la fecundidad de las cónyuges de los migrantes y los no migrantes controlando las características de la

5 Herrick (1965: 81-83) informa el mismo patrón en Santiago, esto es, tasas de participación masculinas casi iguales en todos los grupos de edad, pero tasas mayores entre los inmigrantes femeninos que entre las nativas. 
Gráfica 2

Puerto Rico: Hijos nacidos vivos de mujeres Casadas legalmente con CÓNYUGE PRESENTE, POR STATUS DE MIGRACIÓN DEL MARIDO, EDAD

DE LA MUJER Y STATUS DENTRO DE LA FUERZA DE TRABAJO DEL MARTDO Y DE LA ESPOSA, 1960
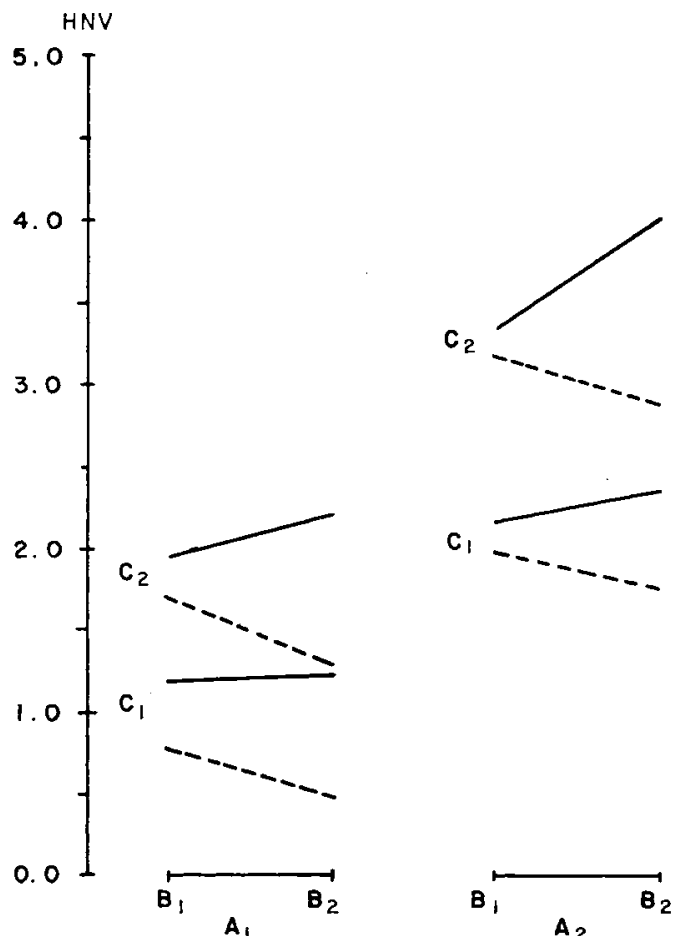

$\mathrm{c}_{2}$
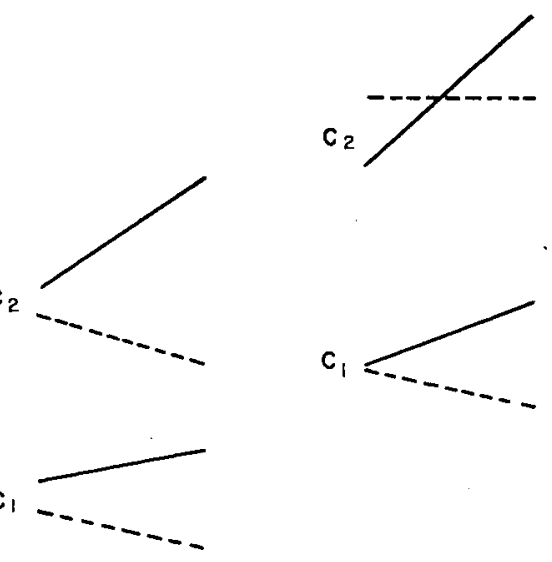

Esposa de migronte

Esposa de no migrante

$A_{1}$, edad de la esposa entre 1424 años; $A_{2}$, edad de la esposa entre 25-34 años; $A_{3}$, edad de la esposa entre $35-44$ años; $B_{1}$, el marido trabaja; $B_{2}$, el marido no trabaja; $C_{1}$, la esposa trabaja; $C_{2}$, la esposa no trabaja; HNV, hijos nacidos vivos.

Cuadro 4

PUERTO RTOO: OCUPACIÓN Y POSTCIÓN MIGRATORIA, AREA EsTadístTCa Metropolitana Estándar de San Juan, 1960

\begin{tabular}{|c|c|c|c|c|c|c|c|c|}
\hline & \multicolumn{4}{|c|}{ Ooupación del marido } & \multicolumn{4}{|c|}{ Ocupacion ae 1 conyuce } \\
\hline & \multicolumn{2}{|c|}{ Migrantes } & \multicolumn{2}{|c|}{ No migrantes } & \multicolumn{2}{|c|}{ Yigranted } & \multicolumn{2}{|c|}{ No mi frantes } \\
\hline & Nitm. & $\bar{x}$ & NLim. & $\%$ & WL.m. & $\%$ & Núm. & $\%$ \\
\hline $\begin{array}{l}\text { Frofesionales y } \\
\text { administraores }\end{array}$ & 229 & 24.4 & 2956 & $24 \cdot 1$ & 117 & 38.4 & 831 & 24.1 \\
\hline $\begin{array}{l}\text { Personal de ofi- } \\
\text { cina } y \text { ventas }\end{array}$ & 105 & 19.7 & 2504 & 20.4 & 88 & 28.9 & 1223 & 35.5 \\
\hline Manual & 494 & 52.7 & 6644 & 54.1 & 99 & 32.5 & 1390 & 40.4 \\
\hline Agricola & 29 & 3.1 & 186 & 1.5 & - & - & - & - \\
\hline TotaI & $937^{2-1}$ & 100.0 & $12290^{\mathrm{b}}$ & 100.0 & $304^{8 / 2}$ & 100.0 & $3444^{\mathrm{d} /}$ & 100.0 \\
\hline
\end{tabular}

Fuente: la misma del cuadro 3.

a Excluye 44 mujeres cuya ocupación no fue declarada.

b Excluye 333 mujeres cuya ocupación no fue declarada.

e Excluye 4 mujeres cuya ocupación no fue declarada.

a Excluye 57 mujeres cuya ocupación no fue declarada. 
Cuadro 5

Puerto Rico: HiJos Nacidos POR CADA 1000 MUjeres CASAdas legalmente, POR EDAD, POSICTÓN OCUPACIONAL DEL MARIDO Y LA ESPOSA Y POSICIÓN MITGRATORIA DEL ESPOSO, 1960

\begin{tabular}{|c|c|c|c|c|}
\hline & $\overline{14-24}$ & \multicolumn{2}{|c|}{ Eded de $2 a$ espose } & $\frac{\text { Total }}{14-44}$ \\
\hline \multicolumn{5}{|l|}{$\begin{array}{l}\text { La espoza y el } \\
\text { marido trabajan }\end{array}$} \\
\hline Esposa de migrante & $\begin{array}{c}775 \\
(79)\end{array}$ & $\begin{array}{l}1980 \\
(144)\end{array}$ & $\begin{array}{r}2816 \\
(76)\end{array}$ & $\begin{array}{l}1904 \\
(291)\end{array}$ \\
\hline $\begin{array}{l}\text { Esposs de no migrant } \\
\text { Cociente } M / \mathrm{rM}\end{array}$ & $\begin{array}{r}1189 \\
(469) \\
.65\end{array}$ & $\left(\begin{array}{rl}2 & 171 \\
1 & 546\end{array}\right)$ & $\begin{array}{r}2871 \\
\left(\begin{array}{r}317 \\
.98\end{array}\right)\end{array}$ & $\begin{array}{r}2312 \\
(3 \quad 324) \\
.82\end{array}$ \\
\hline \multicolumn{5}{|l|}{$\begin{array}{l}\text { La esposa trabaja y el } \\
\text { marido no }\end{array}$} \\
\hline $\begin{array}{l}\text { Esposa de migrante } \\
\text { Esposa de no migrante } \\
\text { Cociente } M / M M\end{array}$ & $\begin{array}{r}500 \\
(6) \\
1280 \\
(25) \\
.39\end{array}$ & $\begin{array}{r}1778 \\
(18) \\
2365 \\
(63) \\
.75\end{array}$ & $\begin{array}{r}2667 \\
(10) \\
3278 \\
(72) \\
.81\end{array}$ & $\begin{array}{r}1786 \\
(34) \\
2,606 \\
(160) \\
.69\end{array}$ \\
\hline \multicolumn{5}{|l|}{$\begin{array}{l}\text { La esposa no trabaja } y \\
\text { el marido of }\end{array}$} \\
\hline $\begin{array}{l}\text { Esposa de migrante } \\
\text { Esposa de no migrante } \\
\text { Cociente } \mathrm{M} / \mathrm{MM}\end{array}$ & $\begin{array}{r}1698 \\
(192) \\
1961 \\
(1902) \\
\quad .87\end{array}$ & $\begin{array}{r}3183 \\
(245) \\
3355 \\
(3 \quad 639) \\
.95\end{array}$ & $\begin{array}{r}4500 \\
(178) \\
4 \quad 101 \\
(3280) \\
1.10\end{array}$ & $\begin{array}{r}3101 \\
(615) \\
3 \quad 364 \\
(8621) \\
.92\end{array}$ \\
\hline \multicolumn{5}{|l|}{$\begin{array}{l}\text { La esposa y el marido } \\
\text { no trabajan }\end{array}$} \\
\hline $\begin{array}{l}\text { Esposa de migrante } \\
\text { Esposa de no migrante } \\
\text { Cociente } \mathrm{M} / \mathrm{MM}\end{array}$ & $\begin{array}{r}1300 \\
(10) \\
2243 \\
(103) \\
.58\end{array}$ & $\begin{array}{r}2918 \\
(19) \\
4016 \\
(128) \\
.73\end{array}$ & $\begin{array}{r}4500 \\
(12) \\
5014 \\
(222) \\
.90\end{array}$ & $\begin{array}{r}3128 \\
(41) \\
4102 \\
(453) \\
.76\end{array}$ \\
\hline
\end{tabular}

Fuente: la misma del cuadro 3 . Número de casos entre paréntesis.

fuerza de trabajo. En la gráfica 2 puede verse que en 11 de 12 comparaciones las esposas de los inmigrantes tienen fecundidad menor que las de los no migrantes. La posición ocupacional del marido no da al parecer lugar a diferencias importantes en relación con la fecundidad, pero parece existir interacción entre la posición ocupacional del marido, la posición migratoria y la fecundidad. Esta interacción es producida de la siguiente manera. Si los no migrantes no están en la fuerza de trabajo, la fecundidad es mayor; pero si los inmigrantes no están en la fuerza de trabajo, entonces la fecundidad es menor. Pero existe un número muy pequeño de casos en algunas de las celdas y resulta incierto si esta interacción observada es real o si es una ilusión estadística.

No obstante, el control de las diferencias en las características ocupacionales no produce la relación positiva esperada entre el origen no metropolitano y la fecundidad. 
Cuadro 6

Puerto Rico: Hijos nacidos por cada 1000 Mujeres casadas legalmente SEGÚN POSICIÓN OCUPACIONAL Y MIGRATORIA, 1960

\begin{tabular}{|c|c|c|}
\hline Posición ocupacional & Hombres & Mujeres \\
\hline \multicolumn{3}{|c|}{ Frofesionales y administrativas } \\
\hline $\begin{array}{l}\text { Migrantes } \\
\text { No migrantes } \\
\text { Cociente } \mathrm{M} / \mathrm{MM}\end{array}$ & $\begin{array}{r}2022 \\
2399 \\
.84\end{array}$ & $\begin{array}{r}1935 \\
1928 \\
1.00\end{array}$ \\
\hline \multicolumn{3}{|l|}{ De oficina $y$ ventas } \\
\hline $\begin{array}{l}\text { Migrantes } \\
\text { No migrantes } \\
\text { Cociente } \mathrm{M} / \mathrm{NM}\end{array}$ & $\begin{array}{r}2244 \\
2548 \\
.88\end{array}$ & $\begin{array}{r}1250 \\
1684 \\
.74\end{array}$ \\
\hline \multicolumn{3}{|l|}{ Manuales } \\
\hline $\begin{array}{l}\text { Migrantes } \\
\text { No migrantes } \\
\text { Cociente } \mathrm{M} / \mathrm{NM}\end{array}$ & $\begin{array}{r}3455 \\
3437 \\
1.01\end{array}$ & $\begin{array}{r}2586 \\
3.056 \\
.84\end{array}$ \\
\hline \multicolumn{3}{|l|}{ hgricolas } \\
\hline $\begin{array}{l}\text { Migrantes } \\
\text { No migrantes } \\
\text { Cociente } \mathrm{M} / \mathrm{NM}\end{array}$ & $\begin{array}{r}4093 \\
4623 \\
.88\end{array}$ & -- \\
\hline
\end{tabular}

Fuente: la misma del cuadro 1.

* Estandarizado en cuanto a composición por edades, en donde la población estándar es el total de la casada legalmente, con esposo presente, femenina, residente en el Área Estadística Metropolitana Estándar de San Juan, en 1960. Las tasas femeninas no están estandarizadas debido a que la distribución de frecuencias en algunos casos lo hace impráctico.

\section{CONCLUSIONES}

A pesar del hecho de que la investigación realizada en las sociedades más industrializadas ha indicado que los residentes metropolitanos con antecedentes no metropolitanos tienen mayor fecundidad que la segunda generación de residentes de la metrópoli, y a pesar de que la fecundidad rural en Puerto Rico (4 533 niños nacidos por cada 1000 mujeres alguna vez casadas de las edades 15-44) es acentuadamente mayor que la fecundidad en el AMSJ ( 3068 niños nacidos) (Oficina del Censo de los Estados Unidos, 1962, pp. 275-276), los inmigrantes de origen no metropolitano no tienen fecundidad mayor que los residentes no migrantes de San Juan. En los grupos de edad más jóvenes su fecundidad es algo menor.

Tal patrón puede ser el producto solamente de la migración selectiva. Tal vez la migración en sí misma es selectiva respecto a fecundidad baja. Podría haber selección por parte de las personas solas y de las familias más pequeñas entre aquellas ya casadas. Debido a la ruptura de sus vidas involucrada en el cambio de comunidades, los migrantes pueden casarse posteriormente a los no migrantes. Así, puede que comiencen a tener hijos a una edad posterior a la de los no migrantes $y$, aun cuando puedan tener una fecundidad marital mayor, su fecundidad, según es medida por una medida acumulativa tal como los hijos nacidos vivos, puede resultar algo menor. También padría ser que, entre aquellos ya casados, mientras menor sea el tamaño de la familia, más fácil debe ser emigrar en vista de los costos económicos menores, la dislocación personal menor y la mayor facilidad para encontrar vivienda aceptable. Sin embargo, la selección de 
este tipo debe estar altamente relacionada con la edad, y el patrón esperado de fecundidad menor entre los migrantes no se presenta en ninguno de los grupos de edad estudiados, y tampoco cuando se calculan medidas estandarizadas en cuanto a la edad. Debemos descontar también la propensión diferencial a las uniones legales versus las consensuales. Aunque el análisis presente se limita a mujeres casadas legalmente los análisis anteriores de Myers y Morris (1966) incluían a mujeres en unión libre y casadas legalmente.

Por lo tanto, debe presentarse igualmente una selección respecto a otras variables. Se ha sugerido que a este respecto pueden estar funcionando variables socioeconómicas, lo cual parece posible, ya que los datos censales de Puerto Rico muestran que los inmigrantes a San Juan han terminado más años de escuela que sus contrapartes no migrantes y con mayor probabilidad tienen esposa que trabaja en una ocupación de "cuello blanco". Cada uno de estos factores tiene una asociación inversa con la fecundidad. Sin embargo, aun cuando controlamos estos factores, los inmigrantes resultan aún sin una fecundidad mayor. Por lo tanto, debe existir selectividad en otros factores además de la edad, el estado civil, la educación y las características ocupacionales.

En la porción restante de este trabajo nos gustaría sugerir otra variable sobre la cual puede presentarse el fenómeno de selección, y citar también dos tipos de investigación adicionales que pensamos son necesarios para entender a fondo la relación entre migración ruralurbana y fecundidad.

El tipo de investigación necesaria es en gran medida metodológico. Deben hacerse comparaciones entre la fecundidad de los nativos urbanos y los inmigrantes de acuerdo con la edad, la generación de residencia en la ciudad, la edad en el momento de la migración hacia la ciudad, la duración de la residencia en la ciudad, el tamaño del área de origen, y sobre si la migración ha sido directa o por etapas. Con tal conocimiento, uno tendría los parámetros de la asociación entre la migración y la fecundidad, aunque no necesariamente sus causas. Un tipo de investigación corolaria es el grado en que varían las características socioeconómicas de acuerdo con cada una de estas distinciones.

Segundo, debe ser fructífero examinar las variaciones en el contexto en el cual ocurre la migración. A este respecto, resulta de la mayor importancia una tipología de contextos. (Balán, 1969, representa un esfuerzo inicial de gran valor.) La investigación de este tipo podría permitir penetrar más sobre por qué en un país se presenta la selección económica de un tipo y en otro no se presenta, y de por qué la relación entre migración y fecundidad parece variar de sociedad a sociedad. Por ejemplo, nos preguntamos si la extensión de las redes de transporte, el ritmo de crecimiento económico, el alcance de la educación formal en las áreas rurales, y la medida de la urbanización tendrían alguna influencia sobre si los migrantes a la ciudad tienen mayor (o menor) fecundidad que los nativos y de si sus características socioeconómicas son o no inferiores. El efecto de la disponibilidad de vivienda y la migración previa de los miembros de la familia y los amigos cercanos que facilitan la obtención de empleo, así como 
los problemas de ajuste a la vida urbana, parecerían también importantes.

Un tipo de investigación final que nos parece debería ser realizada, se refiere a una variable adicional sobre la cual puede existir selectividad. Aunque nuestros datos no son adecuados para llegar a esta noción, nos parece que no son incongruentes con las sugerencias siguientes y pueden servir para aumentar su atendibilidad.

Específicamente, puede ser que una proporción sustancial de los inmigrantes hacia un área metropolitana principal puedan tener más aspiraciones de movilidad social ascendente que la mayoría de los residentes en la ciudad. Si es así, entonces las diferencias en los niveles educativos y en las características ocupacionales podrían verse como síntoma de esta diferencia más básica. De este modo, la migración rural-urbana puede ser selectiva entre las personas con grandes aspiraciones que creen que la economía urbana ofrece mayores oportunidades de movilidad ascendente que lo que permite la estructura social rural estática. Speare (1969) informa que la migración hacia Taichung, Formosa, tiende a ser selectiva respecto a los hombres de mayor educación y que más de la mitad de los inmigrantes se trasladaron para obtener un empleo mejor o porque no había trabajo disponible en el lugar de origen. Esto apoya la noción de que los migrantes rurales urbanos son personas que desean tener éxito y no aceptan en forma pasiva su medio ambiente.

En contraste, los nativos urbanos deben incluir a los que aspiran a tener una movilidad social ascendente y a personas que tienen menos aspiraciones. Con respecto a Puerto Rico, parece probable que los nativos de San Juan con mayores aspiraciones tienden a emigrar a los Estados Unidos. En otras sociedades con menos acceso a otra economía más metropolitana, tales personas pueden simplemente convertirse en migrantes intermetropolitanos. De este modo, las comparaciones del tipo que nosotros y otros demógrafos hemos elaborado son entre inmigrantes y personas que permanecen en la metrópoli. Del mismo modo que los que permanecen en el campo tienen menos aspiraciones que las personas del medio rural que emigran, los que permanecen en la metrópoli pueden tener menos aspiraciones que los que salen de ella. El resultado final puede ser que los inmigrantes tengan mayor orientación hacia el logro y la interacción activa con el medio ambiente que aquellos que permanecen en la metrópoli. ${ }^{6}$

Tal hipótesis ciertamente es consistente con la interacción observada antes entre la posición ocupacional del marido, la posición migratoria y la fecundidad. ${ }^{7}$ Supuestamente, los no migrantes han sido

6 Luis Ramallo, director de FLACSO en Santiago de Chile, afirma en un informe inédito que los migrantes a Asunción muestran una necesidad mayor de logro (según es medido con el método de McClelland), que un grupo de no migrantes nacidos en aquella ciudad. Hasta donde sabemos, éste es el primer intento por probar este tipo de hipótesis. Esto fue comunicado personalmente por Ramallo al segundo de los autores del trabajo presente.

7 Se señaló antes que en algunas casillas del cuadro 5, el número de casos es extremadamente pequeño. Esto sucede cuando el esposo es migrante y no trabaja. Por lo tanto, esta integración observada puede ser un engaño estadístico. Sin embargo, si es real, nos parece que la explicación siguiente es plausible y amerita investigación ulterior. 
expuestos a la oportunidad de recibir la educación y entrenamiento apropiados para participar en la economía metropolitana. Por lo tanto, los que no pertenecen a la fuerza de trabajo son o bien personas que no pueden encontrar empleo permanente o personas muy ricas que no necesitan empleo. En una cultura que favorece la fecundidad alta (frente a las áreas más industrializadas), los últimos deberían tender a tener una fecundidad mayor que las personas con circunstancias socioeconómicas más modestas para quienes el empleo es necesario. La persona incapaz de mantener un empleo constante probablemente tiene también una educación muy limitada y tendería a acusar fecundidad mayor debido a su posición sociocconómica tan baja y también a que no existe razón por la que deba creer que la posición económica de su familia se vería afectada por el número de hijos que tiene. Aun cuando estuviera disponible, el control de la fecundidad puede no verse como necesario o deseable.

En contraste, los migrantes tienden a tener mejor educación que los que permanecen en el área de origen y destino. Más aún, la migración en sí puede tender a ser selectiva en las personas cuya fecundidad inicial es menor. Si el migrante aspira a la movilidad social ascendente y percibe a sus hijos como obstáculo a esta movilidad, entonces su conducta será tal que pospondrá ta procreación hasta que se hayan satisfecho los requisitos de la movilidad. Dos de estos requisitos son la migración al área metropolitana y el acceso consiguiente al mercado de trabajo metropolitano, así como a mantener un empleo constante. Por lo tanto, la persona que emigra y que es incapaz de encontrar empleo fijo puede mostrar grandes deseos por limitar el tamaño de la familia bajo tales circunstancias y posponer la procreación durante un espacio de tiempo mayor que la persona que tiene éxito en obtener empleo y por lo tanto tiene mayores recursos financieros a su disposición. Además, el migrante puede ser en términos generales un mejor planificador, porque ve modos de conducta alternativos, por ejemplo, la residencia en la ciudad vs. la rural y la de tener menos o más hijos. ${ }^{8}$

Por lo tanto, sugerimos un modelo en el cual el matrimonio temprano, la llegada de hijos, la residencia rural y la falta de educación sean considerados como obstáculos a la movilidad social ascendente. Dentro de este contexto, la migración a un centro metropolitano, la edad mayor al matrimonio, la restricción del tamaño de la familia dentro del matrimonio y las elevadas tasas de participación femenina en la fuerza de trabajo, pueden considerarse como respuestas a (o consecuencias de) el deseo de una movilidad social ascendente. Todos estos factores tienden a tener una relación inversa con la fecundidad, y cualquier asociación observada empíricamente entre la migración y la fecundidad es producida indirectamente por estas variables antecedentes. Este modelo es presentado con propósitos heurísticos en

8 La relación entre posición migratoria y conocimientos anticonceptivos es algo relativamente poco explorado. En una comunicación personal con el primero de los autores de este trabajo, George Myers escribe que la investigación sobre fecundidad en varias ciudades emprendida por CELADE indica que los migrantes son motivados a tener menos hijos que los no migrantes, pero les faltan conocimientos sobre cómo lograrlo. Resulta clara la necesidad de mayor investigación en este terreno. 


\section{Gráfica 3}

RELACIONES HIPOTÉTICAS ENTRE MIGRACIÓN, FECUNDIDAD Y ASPIRACIONES DE MOVILIDAD SOCIAL

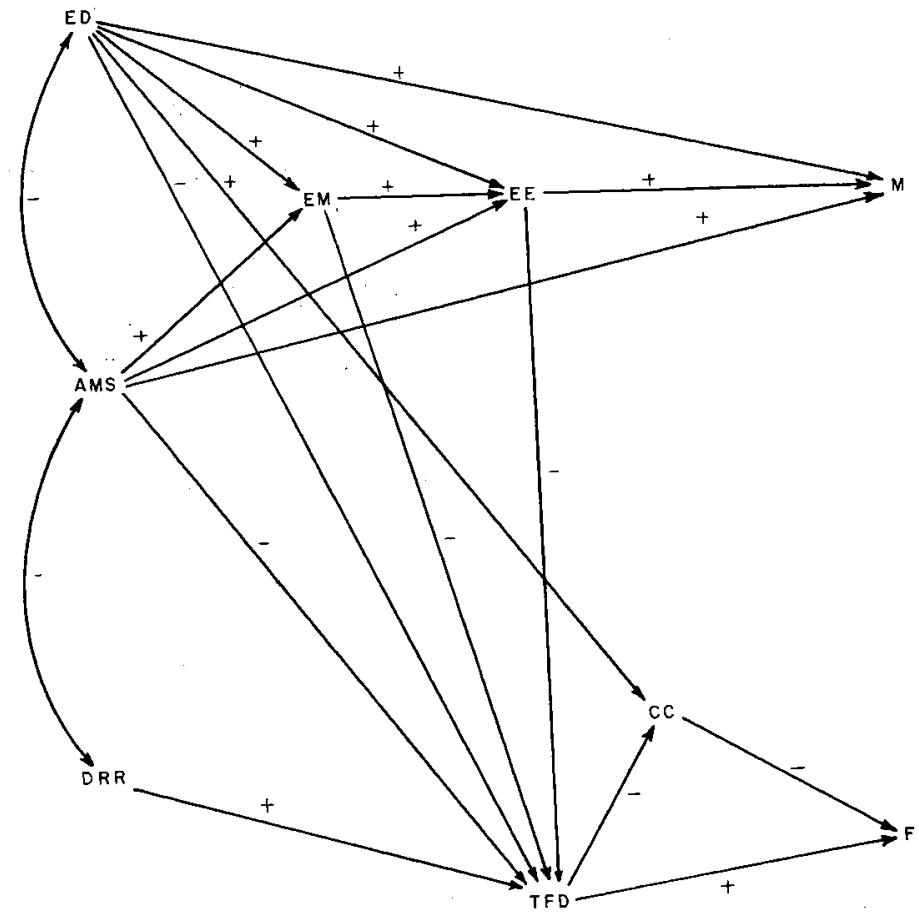

AMS, aspiraciones de movilidad social; CC, conducta contraceptiva; TFD, tamaño de familia deseado; DRR, duración de residencia rural; ED, educación; $F$, fecundidad; M, migración rural urbana; EM, edad al matrimonio; EE, empleo de las esposas.

la gráfica 3. Nos gustaría hacer hincapié en que éste es un modelo hipotético sobre una parte de los migrantes. Aunque creemos que el modelo saldrá favorecido en la prueba empírica, sólo se dispone de evidencia indirecta para porciones significativas del modelo, y esperamos poder probarlo en un futuro próximo.

Reconocemos que los estudios empíricos no han establecido una asociación negativa entre la movilidad social y la fecundidad. Sin embargo, debe señalarse aquí que hay numerosos problemas de medición involucrados que no han sido resueltos en forma satisfactoria. Éstos incluyen la medición de la fecundidad y la determinación de lo que constituye la "movilidad social". Con respecto a lo primero, los demógrafos han tendido a utilizar medidas de fecundidad completa o acumulativa, $y$ no consideran medidas que pongan énfasis en el espaciamiento y el tiempo. Asi, Tien (1970) afirma que la procreación puede ser incompatible con la movilidad desde el punto de vista del tiempo más que de un tamaño eventual de la familia. Perruci (1967; 
1968) ha puesto en duda también lo adecuado del enfoque sobre el tamaño de la familia en lugar del tiempo de ocurrencia de la fecundidad dentro del matrimonio (y el tiempo mismo del matrimonio), como prueba de la hipótesis de la movilidad social y la fecundidad.

Otro punto que debe enfatizarse es que hablamos de aspiraciones de movilidad ascendente $y$ que no existe un isomorfismo entre las propiedades de la movilidad social a la que aspiran y la real. Es decir, no todo aquel que aspira a ser móvil lo es; ni todo aquel que es móvil habrá tenido necesariamente aspiraciones altas de tal movilidad. Por último, las aspiraciones per se pueden influir en la conducta humana y de hecho lo hacen.

Así, mientras que la experiencia reciente de los Estados Unidos puede no apegarse a este modelo, puede ser aún pertinente en países en la etapa de transición del desarrollo que experimentan un largo flujo de migrantes de las áreas rurales. El que esto escribe afirma que la movilidad física puede ser un indicador útil del deseo de movilidad social, en especial entre los grupos más jóvenes de la población. Estos migrantes jóvenes tienen en especial mayor probabilidad de constituir un grupo estratégico ya que a menudo están al margen de los sistemas rurales más tradicionales, y por lo tanto pueden convertirse en innovadores en el complejo metropolitano. ${ }^{9}$

Esta visión de ciertas categorías de migrantes como innovadores no está de acuerdo con la descripción que se ha hecho con frecuencia acerca de las personas desorganizadas que sufren impactos culturales. Se ha juzgado que los inmigrantes exhiben elevada incidencia de patología social y personal como resultado de su incorporación a un nuevo sistema social para el cual están pobremente equipados. En últimas fechas ha surgido otro enfoque al proceso de migración y que desafía a este enfoque del impacto cultural (cf. Cardona 1968; Lewis, 1952; Tilly y Brown, 1967, y Turner, 1963).

Este punto de vista sugiere que la urbanización puede de hecho ocurrir con una desorganización personal mínima y que muchos de los índices observados en la conducta y las características de los migrantes reflejan ajustes funcionales al medio urbano. Sugerimos que en Puerto Rico el migrante entre áreas no metropolitanas y metropolitanas, debido a su mayor educación y al aparente deseo de tener éxito e innovarse, puede igualmente (si no es que mejor) ajustarse a la noción occidental de un medio urbano racional que el no migrante metropolitano. Así, un modelo de movilidad social con consecuencias que conducen a aplazar y limitar la fecundidad puede resultar muy apropiado en países en su etapa de transición del desarrollo. La migración puede jugar un papel principal en la determinación de los niveles de fecundidad urbanos, dependiendo de la magnitud de la corriente migratoria, así como de los niveles de fecundidad de los migrantes y de su descendencia, además de los efectos sobre la tasa

9 Everett Rogers (1962: 172-174) presenta evidencia de que la gente joven tiene mayor propensión a la innovación que los viejos. Emile Durkheim (1964: 295-296) desde luego consideró la concentración de jóvenes en las ciudades que resultó de la migración como una razón primordial de por qué en los centros urbanos se introducen y se aceptan rápidamente las innovaciones mientras que las tradiciones se descartan con facilidad. 
de cambio social. La investigación futura puede explorar con mayor utilidad el papel del inmigrante con antecedentes no metropolitanos como agente innovador en el ambiente metropolitano.

A lo largo de esta sección nos hemos preocupado por el grado en que puede generalizarse el caso de Puerto Rico. Son pocos los casos en que los residentes de un país en desarrollo poseen el mismo grado de acceso a un país metropolitano, económicamente desarrollado, como los Estados Unidos. Así, las personas con aspiraciones y deseos de innovación nacidos en los centros metropolitanos de otros países pueden no ser capaces de emigrar a la misma escala. Esto tendría el efecto de elevar las características de los nativos metropolitanos enumerados en un punto en el tiempo y por lo tanto reduciría las diferencias entre inmigrantes con pasado no metropolitano, y los no migrantes metropolitanos. Sin embargo, también puede suceder que ias personas que aspiran a la movilidad social ascendente en estos países migren entre las metrópolis en un esfuerzo por mejorar su situación.

Parece también poco probable que el modelo establecido en la gráfica 3 pueda generalizarse a todos los migrantes. Tal modelo parecería más aplicable a personas que emigran al centro metropolitano entre las edades 15 y 19 . Si la migración se presenta antes de esa edad, se puede dudar del grado al cual el movimiento es voluntario y por lo tanto el grado al cual las aspiraciones de una movilidad ascendente son la causa de la migración. Si la migración se presenta antes de esa edad, la fecundidad tendería a preceder a la migración y puede inclusive afectar el hecho de que la familia emigre o no.

\section{REFERENCIAS BIBLIOGRAFICAS}

J. Abu-Lughod, "Migrant Adjustment to City Life: The Egyptian Case", American Joturnal of Sociology, 67, julio de 1961, pp. 22-32.

J. O. Alers y R. P. Appelbaum, "La migración en el Perú: Un inventario de proposiciones", Estudios de población y desarrollo, 1, 1968, p. 4, Serie original Núm. 2.

E. E. Arriaga, "Components of City Growth in Selected Latin American Countries", Milbank Memorial Foundation Quarterly, 46, abril de 1968, pp. 237-252.

J. Balán, "Migrant-Native Socioeconomic Differences in Latin American Cities: A Structural Analysis", Latin American Research Review, 4, 1969, pp. 3-29.

D. J. Bogue y M. J. Hagood, Diferential Migration in the Corn and Cotton Belts, Oxford, Scripps Foundation Studies of Population Distribution, Núm. 6, 1953.

L. F. Bouvier y J. J. Macisco Jr., "Education of Husband and Wife and Fertility in Puerto Rico", Social and Economic Studies, 17, marzo de 1968, pp. $49-59$.

H. L. Browning y W. Feindt, "Diferencias entre la población nativa y la migrante en Monterrey", DEMOGRAFÍA Y ECONOMÍA, 2, 1968, pp. 183-204.

_- "Selectividad de migrantes a una metrópoli en un país en desarrollo: Estudio de un caso mexicano", DEMOGRAFÍA y ECONOMÍA, 2, 1969, pp. 186-200.

G. R. Cardona, "Migración, urbanización y marginalidad", en Urbanización y marginalidad, editado por la Asuciación Colombiana de Facultades de Medicina, Bogotá, 1968.

R. M. Dinkel, "Education and Fertility in the United States", en el Vol. IV de World Population Conference, 1965, Naciones Unidas, Nueva York, 1967.

L. Ducoff, "Población migratoria en un área metropolitana de un país en proceso de desarrollo; Informe preliminar sobre un estudio experimental efectuado en El Salvador", Estadística, 20, marzo de 1962, pp. 131-139.

O. D. Duncan, "Farm Background and Differential Fertility", Demography, 2, 1965, pp. 240-249. 
E. Durkheim, The Division of Labor in Society, Nueva York, The Free Press of Glencoe, 1964.

J. C. Elizaga, "A Study on Inmigrations to Greater Santiago (Chile)", Demography, 3, 1966, pp. 353-377.

J. K. Folger y Ch. B. Nam, Education of the American Population, Washington, Oficina de Publicaciones del Gcbierno, 1967.

G. Germani, "Inquiry into the Social Effects of Urbanization in a Working Class Suburb", en Urbanization in Latin America, editado por Philip M. Hauser, Nueva York, International Documents Service, 1961.

F. H. Godley, "La fecundidad y el nivel educacional - Puerto Rico, 1962", Estadística, 26, junio de 1968, pp. 256-284.

D. Goldberg, "The Fertility of Two-Generation Urbanites", Population Studies, 12, marzo de 1959, pp. 214222.

P. K. Hatt, Backgrounds of Human Fertility in Puerto Rico: A Sociological Survey, Princeton, Princeton University Press, 1952.

B. H. Herrick, Urban Migration and Economic Development in Chile, Cambridge, The M. I. T. Press, 1965.

B. Hutchinson, "Fertility, Social Mobility, and Urban Migration in Brazil", Population Studies, 14, marzo de 1961, pp. 182-189.

C. V. Kiser, "Birth Rates Among Rural Migrants in Cities", Mitbank Memorial Foundation Quarterly, 16, octubre de 1938, pp. 369-381.

o. Lewis, "Urbanization Without Breakdown". The Scientific Monthly, 75, julio de 1952, pp. 31-41.

J. J. Macisco Jr., "Fertility in Puerto Rico: An Ecological Study", Sociological Analysis, 26, otoño de 1965, pp. 157-164.

, L. F. Bouvier, y M. J. Renzi, "Migration Status, Education and Fertility in Puerto Rico, 1960", Milbank Memorial Foundation Quarterly, 47, abril de 1969, pp. 167-187.

_- L. F. Bouvier y R. H. Weller, "The Effect of Labor Force Participation on the Relationship Between Migration and Fertility in San Juan, Puerto Rico", Milbank Memorial Foundation Quarterly, 48, enero de 1970, pp. 51-70.

G. R. Martine, Internal Migration and Its Consequences: The Case of Guanabara State, Disertación doctoral no publicada, Brown University, 1969.

G. Masnick, "Employment Status and Retrospective and Prospective Migration in the United States", Demography, 5, 1968, pp. 79-85.

A. R. Miller, "Migration Differentials in Labor Force Participation: United States, $1960^{\prime \prime}$, Demography, 3, 1966, pp. 58-67.

C. A. Miró, y W. Mertens, "Influences Affecting Fertility in Urban and Rural Latin America", Milbank Memorial Foundation Quarterly, 46, julio de 1968, pp. 89-117.

, y F. Rath, "Preliminary Findings of Comparative Fertility Surveys in Three Latin America Countries", Milbank Memorial Foundation Quarterly, 43, actubre de 1965 , pp. $36-62$.

G. C. Myers y E. W. Morris, "Migration and Fertility in Puerto Rico", Population Studies, 20, julio de 1966, pp. 85-96.

G. R. Orbe, R. T. Caicedo, y A. Costales, "Problems Confronting the City-Planner and Administrator in the Town of Esmeraldas, Ecuador", en Urbanization in Latin America, editado por Philip M. Hauser, Nueva York, International Documents Service, 1961.

C. C. Perucci, "Social Origins, Mobility Patterns and Fertility", American Sociological Review, 32, agosto de 1967, pp. 615-625.

-, "Mobility, Marriage and Child-Spacing Among Coilege Graduates", Journal of Marriage and the Family, 30, mayo de 1968, pp. 273-282.

E. M. Rogers, Diffusion of Innovations, Nueva York, Free Press of Glencoe, 1962.

A. Speare, Jr., "The Determinants of Rural-Urban Migration in Taiwan", trabajo presentado en la reunión anual de la Asociación Americana de Población, 1969.

J. M. Stycos, "Education and Fertility in Puerto Rico", en el Vol. IV de Wortd Population Conference, 1965, Naciones Unidas, Nueva York, 1967.

L. Tabah y R. Samuel, "Preliminary Findings of a Survey on Fertility and Attitudes Toward Family Formation in Santiago, Chile", en Research in Family Planning, editado por Clyde V. Kiser, Princeton, Princeton University Press, 1962. 
D. Thomas, Research Memorandum in Migration Differentials, Nueva York, Social Science Research Council, 1938.

H. Y. Tien, "Comparative Analysis of Fertility Change in Developmental Perspective", trabajo preparado para el 7o. Congreso Mundial de la Asociación Internacional de Sociología, 1970.

c. Tilly y H. C. Brown, "On Uprooting, Kinship and the Auspices of Migration", International Journal of Comparative Sociology, 3, septiembre de 1967, pp. 139-164.

J. Turner, "Dwelling Resources in South America", Architectural Design, 8, agosto de 1963 , pp. 369-380.

Naciones Unidas, Departamento de Asuntos Sociales, "Demographic Aspects of Urbanization in Latin America", en Urbanization in Latin America, editado por Philip M. Hauser, Nueva York, International Documents Service, 1961.

Departamento de Censos, Estados Unidos, U. S. Census of Population: 1960, Vol. IV, Detailed Characteristics, parte 53, Puerto Rico, 1962.

J. Vázquez, The Demographic Evolution of Puerto Rico. Disertación doctoral no publicada, University of Chicago, 1964.

R. H. Weller, "The Employment of Wives, Role Incompatibility and Fertility", Milbank Memorial Foundation Quarterly, 46, octubre de 1968, pp. 507-526.

- J. J. Macisco, Jr. y G. R. Martine, "The Relative Importance of the Components of Urban Growth in Latin America, Demography, 8 de mayo de 1971.

L. Wingo, Jr., "Recent Patterns of Urbanization Among Latin American Countries", Urban Affairs Quarterly, 2, marzo de 1967, pp. 81-109.

L. Wirth, "Urbanism as a Way of Life", American Journal of Sociology, 44, julio de 1938, pp. 1-24.

K. D. Zachariah, "Bombay Migration Study: A Pilot Analysis of Migration to an Asian Metropolis", Demography, 3. 1966, pp. 378-392.

A. O. Zárate, "Community of Origin, Migration and Completed Marital Fertility in Metropolitan Monterrey", trabajo presentado a la reunión anual de la Asociación Americana de Población, 1967.

B. G. Zimmer, "Participation of Migrants in Urban Structures", American Sociological Review, 20, abril de 1955, pp. 218-224.

-, "Farm Background and Urban Participation", American Journal of Socio logy, 56, marzo de 1956 , pp. 470-475. 